\title{
EVALUACiÓN DE LA PRESENCIA DE MUJERES EN GABINETES subnacionales de Argentina (1992-2016)
}

\author{
Assessment of the Presence of Women in Subnational Cabinets of \\ Argentina (1992-2016)
}

\section{TIFFANY D. BARNES}

University of Kentucky, EE. UU.

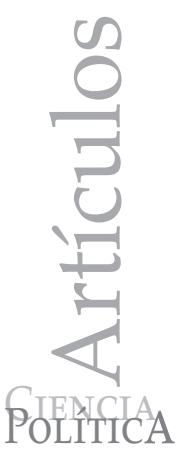

\section{TOMÁS CIOCCI}

Universidad Torcuato Di Tella, Argentina

\section{DÉBORA LOPREITE}

Universidad de Buenos Aires, Argentina

\begin{abstract}
RESUMEN
¿Cuáles son los factores que explican la inclusión de las mujeres en puestos políticos subnacionales? La literatura convencional ha examinado las designaciones de mujeres en gabinetes nacionales, pero escasa atención se ha prestado a los gobiernos provinciales. Para abordar este vacío, adoptamos el enfoque de oferta y demanda. De este modo, utilizando una base de datos sobre las 24 jurisdicciones subnacionales argentinas desde 1992 a 2016, encontramos que los factores del lado de la demanda son más relevantes. Esto se explica, en primer término, ya que la probabilidad de que sean nombradas ministras aumenta junto al tamaño del gabinete, cuando hay una gobernadora mujer $y$, finalmente, si el gobernador pertenece a la coalición peronista. Por otro lado, se ven perjudicadas a medida que aumenta el contingente legislativo del partido del gobernador.
\end{abstract}

Palabras clave: mujeres en gabinetes, representación de mujeres en la política subnacional, liderazgo femenino, género y política subnacional, Argentina

\begin{abstract}
What explains women's inclusion in sub-national cabinet positions? While much of the existing literature has examined women's appointments to national ministerial cabinets, scholars have not considered women's access to provincial level cabinets. To address this gap, we adopt the conventional wisdom of a supply and demand approach to evaluate the findings at the subnational level. Using an original time series dataset from 1992-2016 across all 24 subnational jurisdictions, we find that demand factors are more relevant as the probability that women are appointed ministers increases when cabinets are larger, when there is a female governor, and when the governor belongs to a peronist coalition; conversely, a larger legislative contingent of the governor's party decreases the probability of appointing women.
\end{abstract}

Keywords: women in cabinets, women's subnational representation, women's leadership, gender and subnational politics, Argentina 


\section{INTRODUCCIÓN}

Durante la primera década siglo XXI se registró un acceso sin precedentes de las mujeres a órganos de decisión política en todo el mundo. Este incremento se hizo particularmente evidente en los órganos legislativos debido a la implementación de las cuotas y su impacto positivo en su representación en una gran cantidad de países, especialmente en América Latina. Aun así, es evidente que ellas continúan estando excluidas de los puestos más importantes y prestigiosos dentro de las estructuras gubernamentales, sobre todo en los gabinetes y otros órganos de decisión en donde no existe un mecanismo de discriminación positiva como es el caso mencionado. A pesar de acceder en números nunca antes vistos a las legislaturas, su presencia es escasa o nula en posiciones de liderazgo partidario (O'Brien 2015; Wylie y Dos Santos 2016), en los puestos más poderosos dentro de las legislaturas como la presidencia y vicepresidencia de comisiones legislativas (Schwindt-Bayer 2010; Barnes 2016) y en posiciones de responsabilidad ejecutiva (Escobar-Lemmon y Taylor-Robinson 2016; Jalalzai 2016; Barnes y O’Brien 2018). Las mujeres continúan excluidas de los cargos políticos más poderosos (Barnes 2014; Barnes y Jones 2018), pero también de ámbitos de ejercicio del poder más informales (Franceschet y Piscopo 2014; Barnes 2016).

La investigación académica se ha concentrado en el acceso de las mujeres al poder político, casi exclusivamente cuando se trata de cargos legislativos. Por otro lado, los estudios más recientes sobre su acercamiento a puestos de decisión en gabinetes ejecutivos se enfocan en el nivel nacional (Escobar-Lemmon y Taylor-Robinson 2005, 2016; Krook y O'Brien 2012; Arriola y Johnson 2014; Jalalzai 2016), aunque algunos trabajos más recientes analizan este ingreso a las legislaturas subnacionales en países federales de América Latina (Caminotti et al. 2014; Granara 2014; Barnes 2016; Wylie y Dos Santos 2016; Caminotti y Freindenberg 2016; Escobar-Lemmon y Funk 2018).

Más allá de algunas excepciones (Hinojosa 2012; Holman 2014), la agenda existente ignora en gran medida el acceso de las mujeres a puestos ejecutivos subnacionales, aunque — sobre todo para los países con régimen federal- este tipo de cargos son a menudo más poderosos y codiciados que las posiciones legislativas nacionales (Micozzi 2013). Esto se debe a que estas plazas otorgan acceso a recursos fiscales y poder a los políticos, lo que les permite ejercer una influencia considerable sobre la vida cotidiana de los ciudadanos. La descentralización administrativa y de políticas públicas relevantes, sobre todo en materia social, ha profundizado la autonomía de las provincias y aumentado su poder de decisión, por lo que es crucial comprender las causas que promueven el acceso de las mujeres a cargos en los ministerios provinciales.

Con el objetivo de llenar el vacío de la literatura, ofrecemos un análisis empírico del acceso de las mujeres a los gabinetes subnacionales en Argentina —un país frecuentemente caracterizado como uno de los Estados más descentralizados del mundo (Smith y Revell 2016). Para ello, repasamos algunas de las conclusiones 
encontradas en la literatura especializada que buscan explicar las causas que influyen en el acceso de las mujeres a los cargos del gabinete nacional en América Latina y ver si pueden generalizarse a los gobiernos subnacionales (EscobarLemmon y Taylor-Robinson 2005, 2016). Examinamos dimensiones relativas al sistema político y a los factores socioeconómicos e institucionales que influyen en la presencia de las mujeres en la vida pública, al tiempo que estructuran su acceso a los gabinetes provinciales.

Para testear las hipótesis, utilizamos una base que incluye los gabinetes de las 24 jurisdicciones subnacionales (23 provincias y la Ciudad Autónoma de Buenos Aires, en adelante CABA) para la serie de tiempo 1992-2016. Consideramos que Argentina es un caso relevante por varias razones. En la región es históricamente reconocida como líder en términos de acceso de las mujeres a la política legislativa —particularmente en el ámbito subnacional (Barnes 2016). Como primer país democrático del mundo en adoptar la cuota, la que se difundió rápidamente entre las provincias, Argentina ofrece una visión de largo plazo para estudiar la integración de las mujeres en la política legislativa y, por tanto, se hace relevante estudiar si esta incorporación se trasladó a otros ámbitos institucionales como el poder ejecutivo, teniendo en cuenta que los cargos subnacionales ejercen una influencia considerable sobre las políticas y la asignación presupuestaria.

En la sección que sigue explicamos la importancia de estudiar las causas que explican el acceso de las mujeres a los puestos de gabinete a nivel subnacional. Luego, siguiendo la teoría de Escobar-Lemmon y Taylor-Robinson (2005), examinamos su ingreso a los puestos de gabinete en América Latina y redefinimos algunas variables utilizadas para el presente caso de estudio. La tercera sección describe el método, las variables y los datos empleados mientras que la cuarta presenta la evidencia empírica. En el apartado final se discuten los principales hallazgos y se presentan las conclusiones.

\section{LA RELEVANCIA DE LA PRESENCIA DE MUJERES EN GABINETES MINISTERIALES}

El poder ejecutivo es la rama más prestigiosa y poderosa del gobierno en la que, históricamente, los varones han ocupado la mayoría de los cargos (Jalalzai 2016). A pesar de la creencia actualmente compartida en las democracias de que el poder ejecutivo debe ser el órgano más representativo de la población (Borrelli 2002), el acceso de las mujeres a los cargos ministeriales varía drásticamente, tanto en el tiempo como a través de los países. En América Latina, algunos gobiernos han implementado recientemente gabinetes de paridad, designando en partes iguales a mujeres y varones — como por ejemplo Bolivia y Chile-, aunque en muchos países las mujeres siguen estando escasamente representadas en los gabinetes de los ejecutivos, ocupando solo una pequeña proporción de carteras ministeriales. 
Dado que cada vez más la literatura académica reconoce la concentración del poder político y económico en los poderes ejecutivos, un creciente número de investigaciones intentan explicar la variación del acceso de mujeres a cargos ministeriales (Siaroff 2000; Bauer y Tremblay 2011; Krook y O’Brien 2012; Arriola y Johnson 2014; Claveria 2014). Sin embargo, estas se enfocan casi exclusivamente en los nombramientos en los ejecutivos nacionales, al tiempo que la tendencia global a la descentralización política y administrativa aumenta el poder político y económico de los gobiernos subnacionales, haciendo necesario considerar el acceso de las mujeres a puestos de decisión ejecutiva.

En primer lugar, los gobiernos subnacionales constituyen un ámbito crucial para la competencia política y, por lo tanto, una arena relevante para las mujeres. En muchos regímenes federales, sus cargos ejecutivos subnacionales son más codiciados políticamente que los cargos legislativos —e incluso más que las bancas legislativas nacionales (Micozzi 2013). De hecho, muchos políticos usan a menudo su posición en el congreso nacional como plataforma de lanzamiento al gabinete provincial. Además, las posiciones en los gabinetes subnacionales son vistas como el camino que facilitaría la competencia por la gobernación y, en última instancia, por la presidencia.

No solo las posiciones ministeriales son políticamente valiosas, sino que también dan influencia económica y política significativa a los políticos. El poder ejecutivo provincial es responsable de administrar los recursos fiscales federales y mantiene un alto grado de discreción sobre el gasto provincial, en particular para implementar políticas públicas socialmente relevantes que demandan grandes cantidades de dinero (Bonvecchi y Lodola 2011). Por lo tanto, el acceso de las mujeres a este poder es necesario para que ejerzan su influencia sobre los resultados de las políticas de manera más general, pero es fundamental para la representación sustantiva en cuestiones que influyen desproporcionadamente en las vidas de este segmento de la población. Por ejemplo, los gobiernos subnacionales tienen jurisdicción sobre las políticas de salud, educación y de asistencia social, además de una influencia considerable sobre políticas de género relevantes como aquellas que se oponen a la violencia contra las mujeres y derechos reproductivos (Franceschet 2011; Lopreite 2014; Smulovitz 2015).

Finalmente, el acceso de las mujeres a los ministerios tiene poderosos efectos simbólicos. En el nivel nacional, Morgan y Buice (2013) muestran que, en América Latina, los hombres clasifican la capacidad de las mujeres para gobernar más favorablemente cuando ellas ocupan una mayor cantidad de carteras ministeriales. Barnes y Taylor-Robinson (2018) encuentran que la aprobación y confianza en el gobierno de mujeres y varones impacta positivamente en los nombramientos de estas en los puestos más prestigiosos del gabinete - por ejemplo, defensa, finanzas y asuntos exteriores. Del mismo modo, el aumento de la representación numérica de las mujeres en los gabinetes nacionales se asocia con una menor brecha de género en la participación política (Liu y Banaszak 2017). En el nivel subnacional en particular, Barnes y Jones (2018) demuestran 
que el aumento de la representación numérica de las mujeres en los gabinetes se asocia con mayores niveles de participación ciudadana entre ellas, ya que son más propensas a contactar a su gobierno local cuando hay mujeres en el gabinete provincial.

\section{LA DESIGNACIÓN DE MUJERES EN GABINETES PROVINCIALES: UNA EXPLICACIÓN}

La mayor parte de la literatura se articula alrededor de los factores convencionalmente aceptados- que determinan la oferta y demanda de nombramientos de mujeres en posiciones de liderazgo ejecutivo (Norris 1997; Escobar-Lemmon y Taylor-Robinson 2005). La oferta y la demanda potenciales de ministras están determinadas, en parte, por las preferencias de las mujeres en cuanto a seguir una carrera política. Sin embargo, mujeres y varones comparten la ambición política de acceder a puestos de gabinete con el fin de influir en las políticas y ejercer el control sobre recursos claves, como el presupuesto. Norris (1997) desarrolló un marco de análisis para Europa y Escobar-Lemmon y Taylor-Robinson (2005) lo adaptaron para América Latina. Sin embargo, existen importantes diferencias entre ambas regiones, las que son relevantes para su aplicación en el nivel subnacional.

En este artículo realizamos ajustes en cuanto a la selección de las variables, teniendo en cuenta su aplicabilidad a la realidad subnacional de Argentina.

\section{Mujeres en los gabinetes: análisis de la oferta}

Los componentes del lado de la oferta incluyen las motivaciones del candidato y el capital político, los que están determinados por la experiencia política, los recursos financieros, la ocupación, las calificaciones educativas y las habilidades legislativas. Una primera hipótesis de la literatura convencional refiere al aumento del número de parlamentarias y su impacto en la cantidad de mujeres disponibles que pueden ser nombradas para cargos en el gabinete. Haber contado con puestos electivos — por ejemplo en la legislatura-, puede ser una de las principales calificaciones para obtener nombramientos en el gabinete (Thiébault 1991). En sistemas parlamentarios, los ministros son a menudo reclutados por el Congreso, por lo que el acceso de las mujeres alimenta directamente la disponibilidad de candidatas potenciales para el gabinete (Whitford et al. 2007). Por el contrario, en los sistemas presidencialistas, los ministros no necesariamente son reclutados en la legislatura y, por tanto, el aumento de la representación de las mujeres en el Congreso no necesariamente incrementaría sus chances de ser designadas en cargos ministeriales. Esta misma situación se aplica a los gabinetes provinciales en Argentina, donde rige la separación de poderes. 
Sin embargo, Escobar-Lemmon y Taylor-Robinson (2005) sugieren que la presencia de mujeres legisladoras en América Latina aumenta el número de candidatas, pero ellas lo relacionan con cargos ejecutivos de alto prestigio. Esto sería así porque la experiencia legislativa está asociada con un acceso más amplio a la de tipo político y, por lo tanto, una mejor valoración para los cargos de mayor prestigio del gabinete. Del mismo modo, la presencia de mujeres en instituciones legislativas puede tener un impacto simbólico significativo, ya que naturaliza su presencia en la toma de decisiones y las posiciona mejor para ocupar cargos de alto prestigio ejecutivo (Barnes y O'Brien 2018). Dado que no encontramos una teoría clara para nuestro caso, en todos los modelos utilizamos presencia de mujeres en la legislatura provincial como variable de control.

Ahora bien, la presencia sostenida de mujeres en política aumenta la posibilidad de obtener nombramientos en cargos ejecutivos, esto en relación con la dimensión simbólica de la representación. En este sentido, EscobarLemmon y Taylor-Robinson (2009) señalan que el desempeño de mujeres pioneras - y referentes de la participación femenina en la política- es un factor relevante. Cuando ellas ocupan cargos políticos por un período de tiempo más prolongado, los ciudadanos se acostumbrarían más a que estas ocupen puestos importantes y, por lo tanto, tiene un efecto positivo sobre su presencia en labores de trascendencia para la toma de decisiones, creando conjuntamente un irreversible proceso de cambio en las actitudes y expectativas acerca del rol de las mujeres en política (Schwindt-Bayer 2010). Además, la literatura sobre cuotas de género señala que su efecto simbólico induciría un cambio positivo en la cultura, las normas y las actitudes hacia la representación de las mujeres en la política (Franceschet et al. 2012). La suposición es que esto podría aumentar no solo la oferta de mujeres calificadas en política, sino también la demanda de una mayor inclusión de estas en puestos de liderazgo. Por lo tanto, las cuotas podrían tener un "efecto de aceleración" positivo en el acceso de las mujeres a los puestos políticos más importantes.

H1. La proporción de mujeres en los gabinetes provinciales aumenta directamente con los años a partir de la inclusión de estas en las instituciones representativas.

Además de los factores políticos que aumentan la oferta de mujeres para ocupar cargos en el gabinete, la ampliación de la participación laboral puede aumentar aún más la oferta de candidatas. Debido a que - a diferencia de los cargos legislativos- los cargos ministeriales requieren más frecuentemente de una mezcla de habilidad política y capacitación profesional, la literatura concluye que el aumento de la educación y el porcentaje de mujeres en la fuerza laboral tiene un efecto positivo en su actividad política (Htun y Jones 2001). Además, cuanto más desarrollada y educada sea una población, más receptiva será a las ideas sobre la igualdad de género y la presencia de la mujer en la política y la vida social.

Las hipótesis explicadas en el párrafo anterior, sin embargo, no arrojaron resultados positivos para América Latina. Inesperadamente, Escobar-Lemmon 
y Taylor-Robinson (2005) encontraron que, en los gabinetes nacionales de esta región, si bien solo la educación femenina es significativa, esta es inesperadamente negativa. Asimismo, otros factores socioeconómicos como su participación en la fuerza de trabajo y el grado de educación de la sociedad no afectan los nombramientos de mujeres en dichos cargos. Aunque los hallazgos en la literatura no arrojan resultados unívocos, evaluamos la participación laboral y la educación de las mujeres probando las siguientes hipótesis:

H2. La proporción de mujeres en los gabinetes provinciales aumentará a medida que se incremente el porcentaje de estas en la fuerza laboral.

H3. La proporción de mujeres en los gabinetes provinciales aumentará a medida que se incremente el porcentaje de estas con educación terciaria o más.

\section{Mujeres en los gabinetes: análisis de la demanda}

La demanda de mujeres por ocupar cargos ejecutivos depende de las consideraciones políticas acerca del costo y los beneficios de su designación. De esta manera, los factores por el lado de la demanda determinan las decisiones de los políticos responsables de elegir y nombrar ministros en el gabinete. Por ejemplo, los factores internacionales pueden crear incentivos domésticos por nombrar mujeres debido a la adhesión a convenios internacionales, la presión de organizaciones de mujeres y los posicionamientos de los líderes a nivel regional y global (Jacob et al.2014). De manera similar, en el nivel subnacional son relevantes una serie de factores relacionados con la demanda, así como las medidas previas adoptadas a nivel nacional en materia de derechos y equidad de género.

La literatura comparada a nivel nacional concluye que las mujeres a cargo del ejecutivo son más propensas que sus homólogos masculinos a designar mujeres en sus gabinetes (O’Brien et al. 2015; Reyes-Housholder 2016), lo que lleva a considerar el género del primer mandatario. Esta relación observada no es sorprendente, dado que la investigación feminista argumenta que las mujeres desempeñan un papel fundamental en las actividades como mentoras y apoyo a sus colegas mujeres (Eagly et al. 2003; Barnes 2014). Además, los estudios sobre las percepciones psicológicas del liderazgo demuestran que hombres y mujeres evalúan de manera diferente los atributos para el liderazgo según el género de los candidatos (Norris y Wylie 1995; Deal y Stevenson 1998). Tomados en conjunto, los hallazgos de las investigaciones previas sugieren que una gobernadora puede tener un efecto positivo en la composición de los gabinetes ministeriales según el sexo de los candidatos potenciales para el cargo. Probamos la siguiente hipótesis:

H4. La proporción de mujeres en los gabinetes provinciales aumentará cuando el gobernador sea una mujer. 
Al primer factor de tipo "comportamiento", agregamos otros "institucionales" que pueden influir en las decisiones de nombrar mujeres en los gabinetes: el tamaño del gabinete, el poder legislativo del gobernador y su identificación partidaria.

A medida que aumenta el volumen del gabinete, el grado de competitividad para acceder a estas posiciones puede disminuir, creando así más oportunidades para las mujeres. Esto puede deberse al hecho de que, una vez que los puestos críticos se distribuyen entre los líderes del partido — generalmente hombres-, las carteras políticamente menos relevantes podrían satisfacer el interés de apelar a la diversidad y la inclusión dentro del gabinete. Por lo expuesto,

H5. La proporción de mujeres en gabinetes provinciales aumentará a medida que aumente el tamaño del gabinete.

Otra consideración en la literatura convencional sobre gabinetes nacionales en América Latina se refiere al sistema de partidos y su efecto en los cálculos presidenciales para formar su gabinete vis a vis con su bloque en la legislatura (Reynolds 1999; Escobar-Lemmon y Taylor Robinson 2005). Por una parte, el apoyo que el gobernador tenga en la legislatura puede afectar el costo de elegir mujeres en los cargos ejecutivos, ya que, si tiene mayoría legislativa, no necesita formar coaliciones para la sanción de las leyes. Así, puede utilizar esos cargos para designar a miembros de otros grupos o mujeres si fuese de su preferencia (Franceschet et al. 2017). Este podría ser el caso de San Luis (Argentina), provincia que más mujeres ha tenido en puestos de gabinete y, al mismo tiempo, los oficialismos suelen tener un amplio poder. En el período entre 1992 y 2016, durante un solo año el gobernador no tuvo mayoría legislativa en la Cámara de Diputados, dato acompañado por una media de participación femenina del $32 \%$ en todo el período. Incluso, en los períodos 2001-2003 y 2009-2011, más de la mitad de los ministerios fueron conducidos por mujeres. Solo en dos de los 25 años del período analizado (1996-1998), el gabinete se conformó sin participación femenina.

Por el contrario, un gobernador sin mayoría en la legislatura desearía ofrecer esos cargos a miembros de otros partidos o facciones - usualmente hombrespara ampliar su base legislativa. Este podría ser el caso de la provincia de Buenos Aires, especialmente desde octubre de 2015, cuya gobernadora gana las elecciones mediante una alianza electoral y sin mayoría en la legislatura, tras 28 años de hegemonía del Partido Justicialista. En este contexto, solo una mujer proveniente de una ONG fue designada ministra, quien, sin embargo, duraría un año en el cargo. Por el contrario, varios políticos varones, todos encumbrados dirigentes de terceros partidos, fueron parte del nuevo gobierno: al menos cuatro de partidos opositores a la coalición gobernante y dos ministros pertenecientes a socios minoritarios de la coalición.

De este modo, la hipótesis queda expresada así: 
H6. La proporción de mujeres en gabinetes provinciales aumentará si el gobernador tiene un bloque mayoritario en la legislatura.

Finalmente, en la literatura comparada de género y política se ha señalado ampliamente el efecto positivo que los líderes y partidos de izquierda y progresistas tienen sobre el acceso de las mujeres a puestos de decisión (Caul 2001) $)^{1}$. En Argentina, históricamente, los partidos no reprodujeron la alineación izquierda-derecha más típica de los sistemas partidarios europeos, situación que se reproduce a nivel provincial. Sin embargo, el peronismo ha sido precursor en incorporar mujeres en puestos relevantes de la política - tanto formales como informales. No existe literatura que analice esta relación, pero sí evidencia histórica que sugiere una tendencia del peronismo por reclutar mujeres con alto perfil en sus filas. Esto es así desde la irrupción de Eva Perón como una fuerte Primera Dama en la política argentina en la década del 40 y con la creación de la rama femenina del Partido Peronista (Barry 2009). El peronismo se ha destacado por contar con primeras damas de alto perfil, integrar a una mujer en la fórmula presidencial por primera vez en 1973, elegir a la primera Presidenta de la República en la historia argentina en 2007 y contar con mayor cantidad de mujeres gobernadoras. De este modo, la hipótesis queda expresada así:

H7, La proporción de mujeres en gabinetes provinciales aumentará cuando el gobernador sea del partido peronista.

\section{La designación de mujeres en los ministerios de las provincias argentinas}

Con el objetivo de colocar nuestro estudio de los gabinetes subnacionales en el contexto más amplio del sistema político argentino, realizamos una breve descripción de la situación de las mujeres en la política del país. Como ha sido ampliamente estudiado, en las últimas décadas, la representación política de las mujeres ha aumentado en Argentina debido a la adopción de la Ley de Cuotas legislativas (1991) que estipula un mínimo de 30\% en las listas de candidatos al Congreso Nacional. Las provincias argentinas siguieron esta tendencia, adoptando cuotas legislativas en diferentes momentos, aunque la representación de las mujeres en las legislaturas provinciales está muy por debajo de la del Congreso Nacional. Mientras que en 1983 la representación de las mujeres en las legislaturas provinciales alcanzó solo el 3,2\%, en 2013 esta se incrementó a un 15,03\%, con evidentes variaciones interprovinciales. En 2017 el Congreso argentino sancionó la ley de paridad que estipula que, en las elecciones de 2019, las listas deberán integrar un 50\% de mujeres y50 \% de varones.

Argentina ha tenido y tiene mujeres políticas destacadas. En 2007, Cristina Fernández de Kirchner se convirtió en la primera mujer elegida para la

Aunque otras investigaciones también sugieren que el efecto de la ideología se atenúa con el paso del tiempo y, por lo tanto, aumenta la incorporación de mujeres entre partidos y tendencias conservadoras (Baldez 2002, Htun 2003), el efecto cuota también puede ser matizado (Escobar-Lemmon y Taylor-Robinson 2005). 
Presidencia. Varias candidatas prominentes se postularon para ese cargo ese mismo año. Elisa Carrió, líder de Coalición Cívica (CC), un partido de centroizquierda moderado en ese momento; Vilma Ripol, candidata del Movimiento Socialista de los Trabajadores (MST), conglomerado de izquierda; y la misma Cristina Fernández de Kirchner, candidata del oficialista Frente para la Victoria (FPV), quien obtuvo el 45,29 \% de los votos. Desde 2015, la vicepresidencia es ocupada por Gabriela Michetti y María Eugenia Vidal se convirtió en la primera mujer en gobernar la Provincia de Buenos Aires y derrotar al peronismo desde el retorno a la democracia en 1983, ambas del partido Propuesta Republicana (Pro), con perfil de centro-derecha. Además, en la última elección de medio término del 2017, varias mujeres fueron cabezas de lista para cargos a las Cámaras de Diputados y Senadores, sobre todo en distritos claves como la Ciudad Autónoma de Buenos Aires (CABA) y Provincia de Buenos Aires.

La evolución de la representación femenina en puestos ministeriales nacionales ha mostrado un aumento gradual en la última década (Barnes y Jones 2018). Mientras el 12\% del gabinete durante la presidencia de Fernando de la Rúa (1999-2001) fueron dirigidos por mujeres, los gabinetes de los presidentes Eduardo Duhalde (2002-2003) y Néstor Kirchner (2003-2007), por su parte, mantuvieron un nivel relativamente constante de representación femenina (22-23\%). Sin embargo, la presencia femenina ha disminuido en los últimos años. Según los datos del Instituto Nacional de Estadística y Censos de la República Argentina (Indec), el nombramiento de las mujeres en la oficina ejecutiva nacional alcanzó su pico más alto del 33\% durante el período presidencial 2003-2007. Disminuyó al 25\% durante el período presidencial 2007-2011 y al 20\% durante el período 2011-2015, bajo las presidencias de Cristina Fernández. Finalmente, bajo la presidencia de Mauricio Macri, solo el 9\% de los ministerios están en manos de mujeres.

En las provincias argentinas, las mujeres elegidas para los puestos ejecutivos más altos también permanecen bajas. En 2001, la vicegobernadora de San Luis, Alicia Lemme, reemplazó al gobernador Adolfo Rodríguez Saá cuando ocupó provisionalmente el cargo nacional durante la crisis económica de este país. En 2004, la vicegobernadora de Santiago del Estero, Mercedes "Nina" Aragonés de Juárez, sucedió a su esposo, el gobernador Carlos Juárez, después de su renuncia. En 2007, Fabiana Ríos se convirtió en la primera gobernadora electa del país en Tierra del Fuego. En 2011, Lucía Corpacci fue elegida en Catamarca y en 2013, Claudia Ledesma de Zamora lo fue en Santiago del Estero, quien en 2017 fue sucedida por su esposo, el anterior gobernador de esa provincia. En 2015, María Eugenia Vidal obtuvo la Gobernación de la Provincia de Buenos Aires, mientras que Alicia Kirchner obtuvo la de Santa Cruz y Patricia Bertone la de Tierra del Fuego, respectivamente.

En general, solo el 14\% de las gobernaciones, vicegobernaciones y ministerios están ocupados por mujeres. Sin embargo, entre las provincias, la variación es notable: mientras que Tierra del Fuego y Santiago del Estero se acercaron a la paridad en 2013 — con 45,5\% y 46,2\%, respectivamente-, Entre Ríos nombró una sola mujer en 2016 y después de doce años de gabinetes formados 
exclusivamente por hombres. En este trabajo, intentamos explicar estas variaciones, probando hipótesis desde los enfoques de oferta y demanda de nombramientos de mujeres en los gabinetes provinciales. En la sección que sigue, se presentan los datos y se describen los métodos utilizados para evaluar las hipótesis.

\section{Datos y métodos}

\section{La variable dependiente}

La variable dependiente es la proporción de mujeres que forman parte de los gabinetes en las provincias argentinas. Para medir esto, se reunieron datos sobre la composición total del gabinete para las 23 provincias argentinas y la CABA, desde 1992 a 2016. Los datos incluyen el nombre, el sexo y el ministerio para cada nombramiento durante este período de tiempo ${ }^{2}$. Los 24 años estudiados permiten explorar qué factores se han vuelto más relevantes a lo largo del tiempo. Para ilustrar este cambio, la Figura 1 traza la participación promedio de las ministras en las 24 provincias analizadas en el mencionado periodo de años. Como se observa, en promedio, la presencia de mujeres en los gabinetes ha seguido una tendencia incremental durante el período analizado, con un mínimo de 5\% de estas en el gabinete en 1994. Solo durante el período 20082014, el porcentaje de mujeres en gabinetes se elevó por encima del 15\%.

Figura 1. Porcentaje de mujeres en gabinetes provinciales desde 1992 a 2016

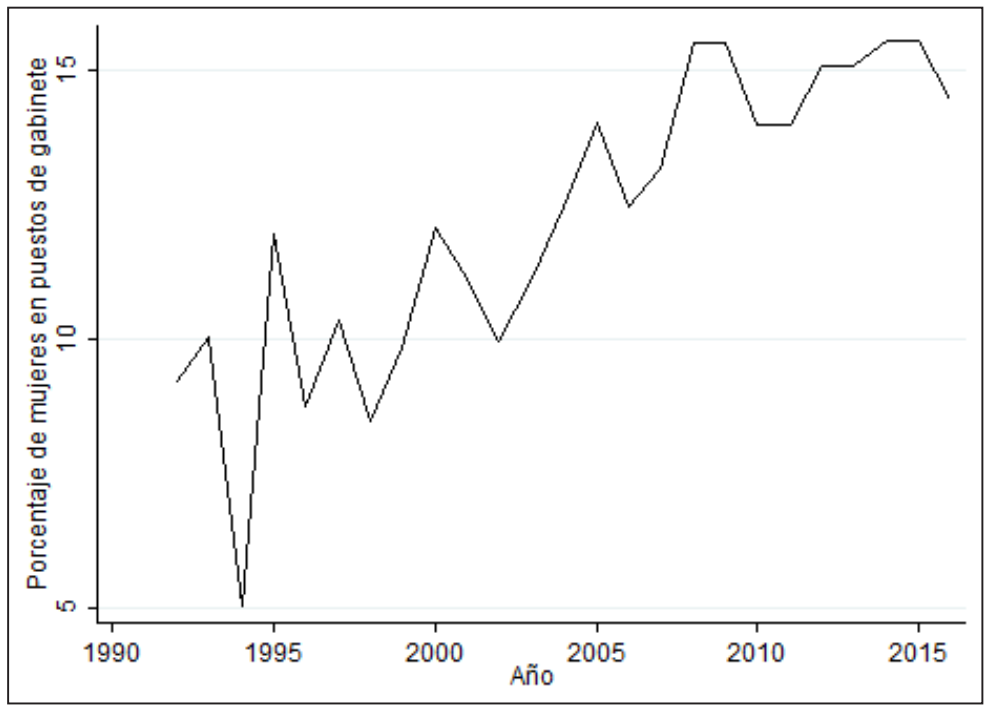

Fuente: Elaboración propia con datos de la base elaborada por Barnes, Ciocci y Lopreite (2018)

2 Los datos se recopilaron principalmente de los boletines oficiales de las provincias. 
A pesar de esta tendencia promedio, existe una variación sustancial entre las provincias. Por ejemplo, las mujeres han tenido una gran cantidad de cargos en varios gabinetes de Argentina, siendo los valores medios para todo el período los siguientes: San Luis (32\%), Santa Cruz (24\%), Mendoza (16\%), Chubut (15\%) y Corrientes (14\%). No obstante, en el resto de las provincias, las mujeres nunca ocuparon más de un $10 \%$ de los puestos del gabinete. Para ilustrar mejor esta variación, la Figura 2 traza la proporción promedio de mujeres en cada provincia durante los 24 años en nuestra muestra.

Figura 2. Media e intervalo de confianza (95\%) de la participación de mujeres por provincia

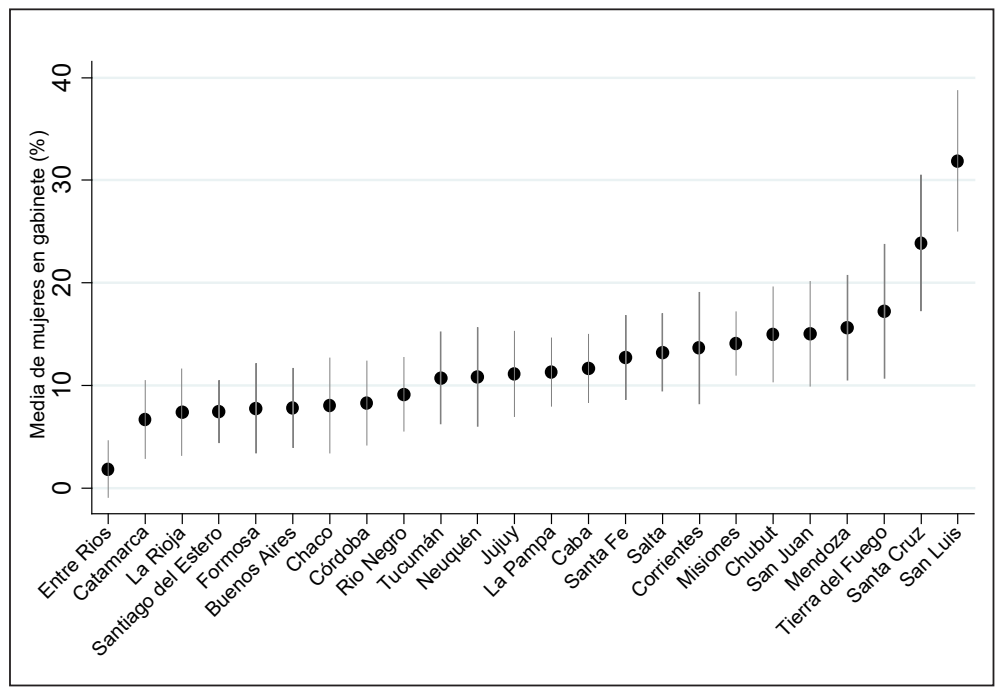

Fuente: Elaboración propia con datos de la base elaborada por Barnes, Ciocci y Lopreite (2018)

\section{Las variables independientes y el modelo de estimación}

En esta sección se describen las variables de oferta y demanda utilizadas para explicar la presencia de mujeres en gabinetes provinciales. En relación a las primeras, la representación de mujeres en la legislatura se midió calculando el porcentaje estas cada dos años, entre 1992 y $2016^{3}$. Elnúmero de años transcurridos desde la inclusión de las mujeres en la política (H1) se mide utilizando la cantidad de años transcurridos desde la promulgación de la Ley de Cuotas en cada provincia. Se entiende que esta medida es un momento significativo en el que, formalmente, se incluyó una masa crítica de mujeres en la política a través de la cuota legislativa. El número de años desde la promulgación de estas leyes en cada provincia tendría diferentes efectos en la representación femenina. 
Además, los años de promulgación de leyes varían mucho entre las provincias, lo que enriquece el análisis. De hecho, mientras algunas provincias adoptaron esta ley en 1992 (Chaco, Corrientes, La Rioja, Santa Cruz, Santa Fe y Chubut), otras implementaron una cuota legislativa en los últimos cuatro años (Jujuy y Entre Ríos). Los años en donde las provincias no habían implementado la cuota legislativa fueron codificados con valor 0 (cero). Como indicador de la presencia femenina en el mercado laboral (H2), utilizamos la población económicamente activa (PEA), medido a partir de la encuesta permanente de hogares (EPH) del INDEC, para el primer trimestre de cada año entre 1992 y 2016. De igual forma, se midió la proporción de mujeres con educación superior en la provincia (H3).

Para evaluar nuestras hipótesis sobre la demanda de mujeres, incluimos las siguientes variables. Un indicador dicotómico sobre el género del gobernador, que fue codificado como 1 si el gobernador es mujer y 0 si es hombre (H4). Para la segunda variable explicativa — tamaño del gabinete (H5)—, se calculó la cantidad de puestos ministeriales en el poder ejecutivo (logaritmo natural), excluyendo los puestos de gobernador y vicegobernador. En tanto el tamaño del bloque del gobernador, se calculó como el porcentaje de bancas en la Cámara de Diputados local (logaritmo natural) pertenecientes a su partido (H6) y, finalmente, se incluyó una variable dicotómica sobre la afiliación partidaria del gobernador, codificada como 1 si el gobernador es del Partido Justicialista (peronismo) y 0 si es de otro partido (H7).

Dada la naturaleza en la composición de la variable dependiente, la regresión por mínimos cuadrados ordinarios (MCO) es inapropiada para probar nuestras hipótesis (Katz y King 1999; Tomz et al. 2002). Para administrar la naturaleza limitada del dato (cada proporción de votos se encuentra entre 0 y 1 ), se utiliza un modelo lineal generalizado (GLM) con una distribución binomial y una función de enlace logístico con errores estándar robustos, que es una solución adecuada para variables dependientes de estas características (Papke y Wooldridge 1996). También se muestra en qué medida los resultados son robustos para el mismo modelo con errores estándar agrupados por provincia y por año, sumado a otros tres modelos en donde se incorporan efectos fijos por año y por provincia, así como por año y provincia al mismo tiempo.

\section{Análisis de los resultados}

La Tabla 1 reporta los coeficientes y errores estándar de nuestros análisis estadísticos. Para evaluar nuestras hipótesis e ilustrar la magnitud de las relaciones, calculamos los valores esperados para la variable dependiente en el rango de las variables independientes, permaneciendo el resto de las variables 
independientes en su valor medio. A su vez, se grafican los resultados para cada una de las hipótesis estadísticamente significativas y se discuten los resultados. ${ }^{4}$

Tabla 1. Explicando la proporción de mujeres en gabinetes provinciales

\begin{tabular}{|c|c|c|c|c|c|c|}
\hline & $\begin{array}{l}\text { (1) } \\
\text { GLM, SE } \\
\text { robustos }\end{array}$ & $\begin{array}{c}(2) \\
\text { GLM, SE } \\
\text { clúster por } \\
\text { provincia }\end{array}$ & $\begin{array}{l}\text { (3) } \\
\text { GLM, SE } \\
\text { clúster por } \\
\text { año }\end{array}$ & $\begin{array}{l}\text { (4) } \\
\text { GLM, SE } \\
\text { robustos }\end{array}$ & $\begin{array}{l}\text { (5) } \\
\text { GLM, SE } \\
\text { robustos }\end{array}$ & $\begin{array}{l}\text { (6) } \\
\text { GLM, SE } \\
\text { robustos }\end{array}$ \\
\hline \multirow[t]{2}{*}{$\begin{array}{l}\text { Mujeres en la } \\
\text { legislatura }\end{array}$} & $-0,00$ & $-0,00$ & $-0,00$ & 0,00 & $-0,01$ & $-0,01$ \\
\hline & $(0,01)$ & $(0,01)$ & $(0,01)$ & $(0,01)$ & $(0,01)$ & $(0,01)$ \\
\hline \multirow{2}{*}{$\begin{array}{l}\text { Años desde } \\
\text { la ley de } \\
\text { cuota }\end{array}$} & $0,02^{*}$ & 0,02 & $0,02^{*}$ & 0,01 & 0,01 & $-0,00$ \\
\hline & $(0,01)$ & $(0,02)$ & $(0,01)$ & $(0,01)$ & $(0,01)$ & $(0,03)$ \\
\hline \multirow{2}{*}{$\begin{array}{l}\text { Mujeres en } \\
\text { el mercado } \\
\text { laboral }\end{array}$} & 0,00 & 0,00 & 0,00 & $-0,00$ & $-0,01^{*}$ & $-0,04^{* * *}$ \\
\hline & $(0,00)$ & $(0,00)$ & $(0,00)$ & $(0,00)$ & $(0,01)$ & $(0,01)$ \\
\hline \multirow{2}{*}{$\begin{array}{l}\text { Nivel edu- } \\
\text { cativo de las } \\
\text { mujeres }\end{array}$} & $-0,02^{*}$ & $-0,02$ & $-0,02$ & $-0,01$ & $-0,02$ & $-0,02$ \\
\hline & $(0,01)$ & $(0,01)$ & $(0,01)$ & $(0,02)$ & $(0,01)$ & $(0,02)$ \\
\hline \multirow[t]{2}{*}{$\begin{array}{l}\text { Género del } \\
\text { gobernador }\end{array}$} & $0,75^{* * *}$ & $0,75^{*}$ & $0,75^{* *}$ & $0,70^{* *}$ & $0,81^{* * *}$ & $0,73^{* * *}$ \\
\hline & $(0,23)$ & $(0,30)$ & $(0,25)$ & $(0,22)$ & $(0,22)$ & $(0,21)$ \\
\hline \multirow[t]{2}{*}{$\begin{array}{l}\text { Log (tamaño } \\
\text { del gabinete) }\end{array}$} & $0,63^{* * *}$ & $0,63^{*}$ & $0,63^{* * *}$ & $0,59 * *$ & $0,72^{* * *}$ & $0,55^{*}$ \\
\hline & $(0,18)$ & $(0,32)$ & $(0,16)$ & $(0,20)$ & $(0,19)$ & $(0,22)$ \\
\hline \multirow{2}{*}{$\begin{array}{l}\text { Log (bloque } \\
\text { del goberna- } \\
\text { dor) }\end{array}$} & $-0,28^{* *}$ & $-0,28$ & $-0,28^{* *}$ & $-0,32^{* *}$ & $-0,38^{* *}$ & $-0,36^{*}$ \\
\hline & $(0,11)$ & $(0,15)$ & $(0,10)$ & $(0,12)$ & $(0,13)$ & $(0,14)$ \\
\hline \multirow[t]{2}{*}{ Peronista } & $0,28^{* *}$ & 0,28 & $0,28^{* *}$ & 0,22 & $0,24^{*}$ & 0,19 \\
\hline & $(0,10)$ & $(0,20)$ & $(0,10)$ & $(0,13)$ & $(0,10)$ & $(0,13)$ \\
\hline \multirow[t]{2}{*}{ Constant } & $-2,08^{* * *}$ & $-2,08^{*}$ & $-2,08^{* * *}$ & $-2,73^{* * *}$ & $-1,12$ & $-0,51$ \\
\hline & $(0,61)$ & $(0,87)$ & $(0,54)$ & $(0,72)$ & $(0,76)$ & $(1,04)$ \\
\hline
\end{tabular}

Para construir los gráficos, transformamos las probabilidades predichas en una escala no logarítmica (con la función exponencial). De esta forma, se pueden entender fácilmente cómo un aumento de una unidad en x (en este caso, el tamaño del gabinete) está asociado con el cambio en la participación de las mujeres en los gabinetes provinciales. Los valores esperados se calculan utilizando el comando predictnl (predicciones no lineales) del menú "postestimation". 


\begin{tabular}{lcccccc}
\hline & $\begin{array}{c}(1) \\
\text { GLM, SE } \\
\text { robustos }\end{array}$ & $\begin{array}{c}(2) \\
\text { GLM, SE } \\
\text { clúster por } \\
\text { provincia }\end{array}$ & $\begin{array}{c}(3) \\
\text { GLM, SE } \\
\text { clúster por } \\
\text { año }\end{array}$ & $\begin{array}{c}\text { GLM, SE } \\
\text { robustos }\end{array}$ & $\begin{array}{c}\text { GLM, SE } \\
\text { robustos }\end{array}$ & $\begin{array}{c}\text { GLM, SE } \\
\text { robustos }\end{array}$ \\
\hline $\begin{array}{l}\text { GLM, EF por } \\
\text { provincia }\end{array}$ & & & & $\sqrt{ }$ & & $\sqrt{ }$ \\
$\begin{array}{l}\text { EF por año } \\
\begin{array}{l}\text { Provincias- } \\
\text { años }\end{array}\end{array}$ & 596 & 596 & 596 & 596 & 596 & $\sqrt{ }$ \\
$\begin{array}{l}\text { Provincias } \\
\text { Años }\end{array}$ & 24 & 24 & 24 & 24 & 24 & 24 \\
\hline
\end{tabular}

Errores estándar entre paréntesis

${ }^{*} \mathrm{p}<, 05,{ }^{* *} \mathrm{p}<, 01,{ }^{* * *} \mathrm{p}<, 001$

\section{Explicando la oferta de mujeres ministras de gabinete}

Ninguna de las variables del lado de la oferta arroja resultados contundentes y significativos. Los años que transcurrieron desde la Ley de Cuotas (H1), la proporción de mujeres en el mercado laboral formal (H2) y aquellas con educación terciaria (H3) muestran significancia estadística de manera aislada y los efectos son cercanos a cero. En relación con nuestra variable de control, los resultados indican que no necesariamente los puestos legislativos en manos de mujeres actúan impulsando el nombramiento de estas en gabinetes.

Para la hipótesis 2 (H2), encontramos que los dos factores no están relacionados. Aunque la investigación en países desarrollados encuentra que la participación laboral de las mujeres es un predictor positivo de la participación política de las mismas (Matland 1998), no ocurre lo mismo en las naciones en desarrollo (Escobar-Lemmon y Taylor-Robinson 2005). Investigaciones previas explican que la fuerza de trabajo femenina en los territorios en desarrollo es más probable que se limite a empleos precarios o de baja calificación, que hacen poco para empoderar a las mujeres e iniciar su participación política de una forma similar a la observada en los estados desarrollados (Matland 1998; EscobarLemmon y Taylor-Robinson 2005). Además, dado que las mujeres en América Latina -incluso las con carreras exitosas- continúan siendo las principales responsables del cuidado de los niños y la familia, la participación de estas en la fuerza de trabajo solo puede contribuir a un número creciente de obligaciones que enfrentan las mujeres. De este modo, si las mujeres tienen carreras exitosas y tienden a realizar tareas domésticas, los nombramientos políticos pueden verse como una carga adicional.

Similarmente a la hipótesis anterior, el mayor nivel educativo de las mujeres no expresa relación significativa. Inglehart y Norris (1993) no encontraron a esta variable como un predictor de mujeres en legislaturas y atribuyen a factores culturales su posible irrelevancia. En efecto, independientemente de la disponibilidad de mujeres educadas y profesionales en Argentina, pueden 
existir barreras culturales basadas en percepciones tradicionalistas respecto de los roles de género que anulen el efecto de la educación para los cargos ejecutivos en las provincias (Inglehart y Norris 1993).

\section{Explicando la demanda de mujeres ministras de gabinete}

Siguiendo con la explicación, se analizan las variables que afectan la demanda de mujeres en el gabinete. La hipótesis 4 (H4) postulaba que las mujeres en el liderazgo ejecutivo darían paso a más nombramientos de estas en el gabinete, a la que se encontró apoyo. Específicamente, la representación numérica de las mujeres es aproximadamente un 10\% más alta en los gabinetes en presencia de una gobernadora. Mientras que el modelo predice que las mujeres tendrán un promedio del $12 \%$ de las carteras bajo el liderazgo masculino, se espera que tengan el $22 \%$ bajo el liderazgo femenino.

Figura 3. Tamaño del gabinete y nombramiento de mujeres en estos

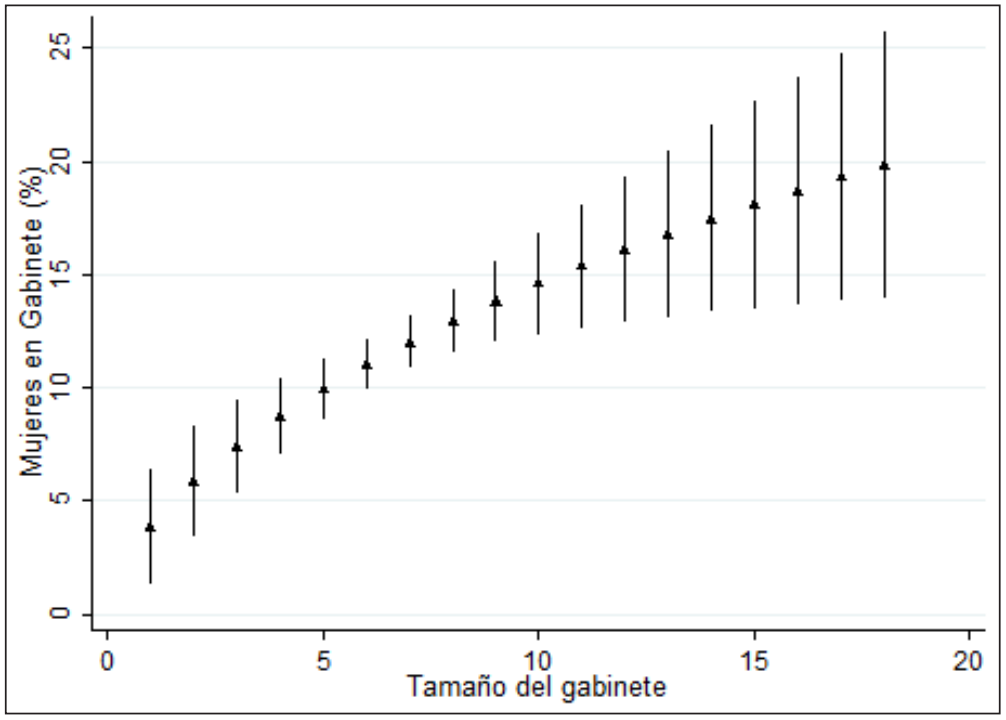

Nota: resultados basados en el modelo 1 de la Tabla 1, el resto de las variables se mantienen en su valor medio.

El aumento en el tamaño de los gabinetes también se asocia con una mayor proporción de nombramientos femeninos como lo sugiere la hipótesis 5 (H5). La Figura 3 ilustra esta relación y, en general, es consistente con los resultados de la literatura comparada citada. Por ejemplo, se espera que las mujeres tengan el $9 \%$ de las carteras en los gabinetes, con una desviación estándar por debajo del tamaño medio (seis ministerios, aproximadamente). Por el contrario, el modelo predice que ellas ocuparán aproximadamente el 15\% de las carteras en los gabinetes, con una desviación estándar por encima del promedio (doce ministerios, aproximadamente). Cuando los gabinetes son 
relativamente pequeños, cada ministerio adicional se asocia con un aumento de aproximadamente un $2 \%$ en la participación de las mujeres. Sin embargo, a medida que el tamaño del gabinete aumenta por encima del promedio, las mujeres ven un rendimiento decreciente en los lugares ocupados.

En el caso de la hipótesis 6 (H6) — que predecía que un gobernador con mayoría legislativa sería más proclive a nombrar mujeres en cargos ejecutivos-, el resultado va en el sentido contrario, es decir, cuando el gobernador cuenta con mayoría es menos propenso a designar mujeres en su gabinete y esta relación es significativa en los seis modelos. Este resultado, visible en la Figura 4, es consistente con parte de la literatura comparada, aunque en este caso es, además, significativo.

Figura 4. Tamaño del bloque del gobernador y nombramientos

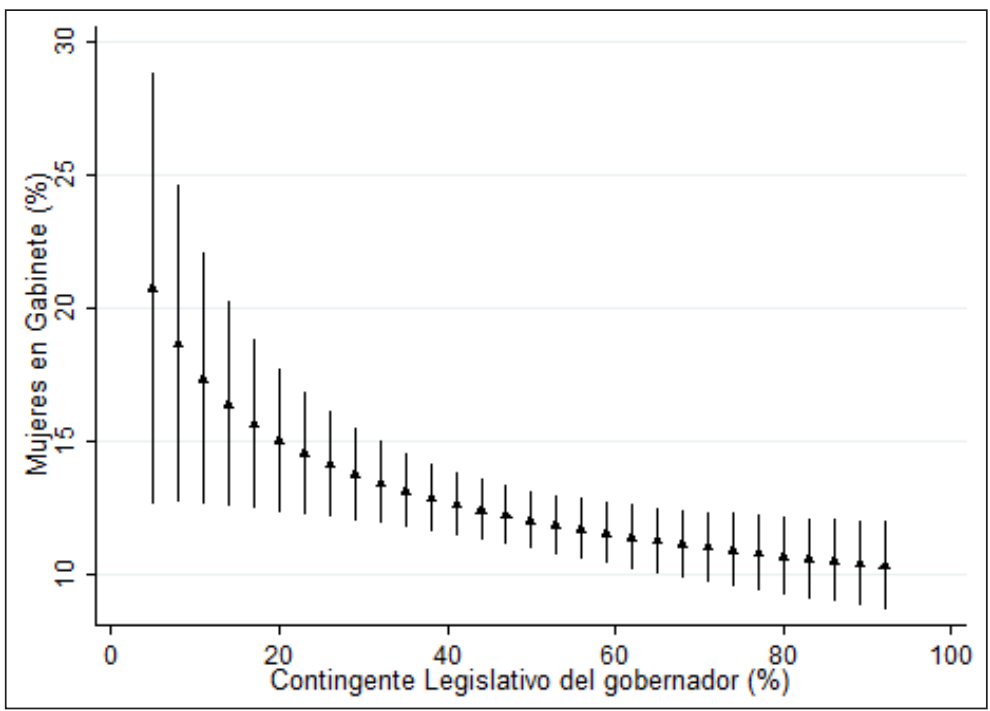

Nota: resultados basados en el modelo 1 de la Tabla 1, el resto de las variables se mantienen en su valor medio.

Una posible explicación se relaciona con el interés del gobernador en ampliar su base de votantes frente a un bloque legislativo minoritario. Caul (2001), por ejemplo, señala que, en Europa occidental, los partidos políticos adoptaron cuotas de género de manera voluntaria como parte de una estrategia competitiva y así atraer el voto de las mujeres. Ciertamente, los primeros partidos en adoptar las cuotas fueron los más pequeños, a los que siguieron aquellos que intentaban recuperar votos de mujeres que habían perdido a expensas de los más proclives a incorporar candidatas en sus listas. Por lo tanto, es posible que los gobernantes con menor apoyo político en las legislaturas adopten una estrategia similar: nombrar a más mujeres en su gabinete en un intento para ampliar su base electoral. Dicho esto, se necesitan más investigaciones para comprender si la 
competencia política es un incentivo positivo para la designación de mujeres en los gabinetes en los mandatos con menos respaldo legislativo.

El resultado de la hipótesis 7 (H7), respecto de la afiliación partidaria del gobernador, es positivo en tres de los seis modelos. Es decir, si el gobernador es peronista, aumenta el porcentaje de mujeres en el gabinete, resultado que es el esperado respecto de la hipótesis planteada. El efecto de la filiación partidaria del gobernador es robusto solo en los modelos que controlan el efecto tiempo y no en el que controla el de la provincia. Esto podría estar sugiriendo que solo algunas provincias, en particular, son más propensas a incorporar mujeres en gabinete, las cuales también han sido históricamente gobernadas por el Partido Justicialista (peronista).

\section{CONCLUSIONES}

Los factores del lado de la demanda demostraron tener efectos significativos en comparación con los de la oferta. Esto sugiere que, en Argentina, los factores que más influyen en el éxito de las mujeres en lograr puestos de gabinete surgen de las demandas del propio sistema político subnacional y no de la disponibilidad relacionada con factores estructurales de las provincias y con la presencia de estas en la actividad política.

Por el lado de la demanda, los Estados más grandes (con gabinetes más grandes) otorgan mayores oportunidades a las mujeres para ocupar ministerios, en consonancia con la literatura convencional. Además, el nombramiento previo de mujeres como gobernadoras demuestra el papel fundamental que ellas desempeñan en el patrocinio de otras carreras femeninas. Esto es consistente con la literatura sobre el liderazgo femenino que señala que las mujeres son más propensas a guiar y apoyar a colegas de su mismo género. Mientras que el género del gobernador es positivo y significativo, la posibilidad de éste de contar con una mayoría legislativa fue negativa y significativa demostrando que, cuantos más legisladores controle el/la gobernador/a menos mujeres nombrará en su gabinete. Esto puede explicarse porque en la mayoría de las provincias de Argentina, las legislaturas son débiles vis a vis los ejecutivos y, por lo tanto, se torna irrelevante la actividad legislativa para la gestión del gobierno y la adopción de políticas que corre por cuenta del ejecutivo. Pero, además, en el caso de un gobierno dividido, esta situación puede incentivar a los líderes a nombrar más mujeres con el objetivo de ampliar su base electoral. Asimismo, la afiliación justicialista (peronista) del gobernador también fue positiva, confirmando la hipótesis de que la tradición de este partido de incorporar mujeres de alto perfil, puede favorecer el acceso de estas a puestos de decisión en los ejecutivos.

En líneas generales, los resultados son consistentes con la literatura comparada y las generalizaciones convencionalmente aceptadas en investigaciones existentes correspondientes a gabinetes nacionales de América Latina. Sin 
embargo, la presente investigación debe ampliarse de varias maneras, sobre todo para ahondar en la especificidad subnacional. Agregar el análisis de los patrones de carreras políticas de mujeres y hombres proporcionará más conocimiento sobre la segregación horizontal de género en las políticas públicas, así como la relevancia de los nombramientos específicos de estas para avanzar en las políticas de igualdad de género y otras políticas sociales relacionadas al cuidado de niños, licencias por maternidad y salud reproductiva, las que están descentralizadas a nivel provincial. Finalmente, los resultados de esta investigación, proporcionarán hipótesis de trabajo para realizar estudios de casos comparativos.

Un caso destacado que surge del análisis de regresión es la provincia de San Luis, donde una proporción muy grande de mujeres son designadas en el gabinete. Este gran número de funcionarias en el gabinete resulta sorprendente frente a la expectativa de que sería más probable que ellas accedan a puestos de mayor responsabilidad en contextos donde predominen valores menos tradicionalistas y más modernos respecto del rol de las mujeres en la sociedad y la vida pública (Holman 2018). Por el contrario, estos resultados sugieren que las mujeres serían más probablemente designadas en puestos ejecutivos cuando la influencia de las dinastías familiares y la vigencia de reglas nepotistas en la política sea mayor. Ciertamente, cuando las cuotas fueron adoptadas en Argentina, fueron vistas como una manera de promover en la política a mujeres "más obedientes" (Franceschet y Piscopo 2008) y a políticas leales (Dahlerup 2006), quienes entonces deberían al partido y a sus lazos familiares su ingreso a la política. Es posible que una dinámica similar se produzca en las provincias donde el gobierno esté controlado por un grupo selecto de líderes poderosos o en aquellas donde esta autoridad esté motivado a mantener mayor control sobre su gabinete.

Otro caso sería el de la Provincia de Buenos Aires, cuya gobernadora, sin embargo, presenta el porcentaje más bajo de mujeres en gabinete en la muestra. En primer lugar, la limitada presencia de mujeres en gabinete resulta sorprendente, ya que se trata de una provincia muy desarrollada, en la que predominan valores menos tradicionales y donde la política es más competitiva. En comparación con San Luis y otras provincias, Buenos Aires tiene una economía más fuerte y diversificada. En provincias con economías más robustas y dinámicas, los cargos ejecutivos son más valiosos y más relevantes para el control de los recursos. Consistente con la literatura que demuestra que las mujeres son generalmente excluidas de las comisiones más prestigiosas y poderosas en las legislaturas (Schwindt-Bayer 2010; Barnes 2016), partidos políticos (O'Brien 2015) y gabinetes (Escobar-Lemmon y Taylor-Robinson 2016; Barnes y O’Brien 2018), es posible que las mujeres sean probablemente designadas en ministerios menos prestigiosos y relevantes para el control de los recursos. La investigación futura debería capturar, más específicamente, la singularidad de la política subnacional argentina a fin de expandir el análisis de los factores que influyen en el acceso de las mujeres a los puestos de decisión de alto perfil y que podrían 
explicar más profundamente la variación en las designaciones de estas a través de las provincias.

\section{REFERENCIAS}

Arriola, Lenardo y Martha C. Johnson. 2014. “Ethnic Politics and Women's Empowerment in Africa: Ministerial Appointments to Executive Cabinets". American Journal of Political Science 58 (2): 495-510.

Baldez, Lisa. 2002. Why Women Protest: Women's Movements in Chile. Cambridge: Cambridge University Press.

Barnes, Tiffany D. 2014. “Women's Representation and Legislative Committee Appointments: The Case of the Argentine Provinces". Revista Uruguaya de Ciencia Politica 23 (2): 135163.

Barnes, Tiffany D. 2016. Gendering Legislative Behavior. New York: Cambridge University Press.

Barnes, Tiffany D. y Diana O’Brien 2018. “Defending the Realm: The Appointment of Female Defense Ministers Worldwide". American Journal of Political Science 62 (2): 355-368.

Barnes, Tiffany D. y Mark Jones 2018. "Women's Representation in the Argentine National and Subnational Governments". En Women, Representation, and Politics in Latin America, editado por Leslie Schwindt-Bayer. New York: Oxford University Press, 121-139.

Barnes, Tiffany D. y Michelle Taylor-Robinson. 2018. "Women Cabinet Ministers and Empowerment of Women: Are the two Related?". En Measuring Women's Political Empowerment across the Globe: Strategies, Challenges and Future Research, editado por Amy Alexander, Catherine Bolzendahl y Farida Jalalzai. Basingstoke, UK: Palgrave Macmillan, 229-255.

Barry, Carolina. 2009. Evita Capitana. El Partido Peronista femenino (1949-1955). Caseros: Editorial de la Universidad Nacional de Tres de Febrero-Eduntref.

Bauer, Gretchen y Manon Tremblay. 2011. Women in Executive Power: A Global Overview. New York: Routledge.

Bonvecchi, Alejandro y Germán Lodola. 2011. "The Dual Logic of Intergovernmental Transfers: Presidents, Governors, and the Politics of Coalition-Building in Argentina". Publius: The Journal of Federalism 41 (2): 179-206.

Borrelli, Mary Anne. 2002. The President's Cabinet: Gender, Power and Representation. Boulder: Lynne Rienner Publishers.

Caminotti, Mariana, Santiago Rotman y Carlos Varetto. 2014. "Desigualdades persistentes. Una mirada a las carreras políticas subnacionales antes y después de las cuotas de género (Argentina, 1983-2011)". En La representación imperfecta. Logros y desafíos de las mujeres politicas, editado por Nélida Archenti y María Inés Tula. Buenos Aires: Eudeba, 201-214.

Caminotti, Mariana y Flavia Freindenberg. 2016. “Federalismo electoral, fortaleza de las cuotas de género y representación política de las mujeres en los ámbitos subnacionales en Argentina y México". Revista Mexicana de Ciencias Políticas y Sociales 61 (228): 121-144.

Caul, Miki. 2001. "Political Parties and Candidate Gender Policies: A Cross-National Study". Journal of Politics 63 (4):1214-1229.

Claveria, Silvia. 2014. "Still a "Male Business"? Explaining Women's Presence in Executive Office". West European Politics 37 (5): 1156-1176.

Dahlerup, Drude. 2006. "The Story of the Theory of Critical Mass". Politics \& Gender 2 (4): 511-22.

Deal, Jennifer J. y Maura Stevenson. 1998. "Perceptions of Female and Male Managers in the 1990s: Plus ça change...". Sex Roles 38 (3-4): 287-300.

Eagly, Alice, Mary Johannesen-Schmidt y Marloes L. van Engen. 2003. "Transformational, Transactional, and Laissez-Faire Leadership Styles: A Meta-Analysis Comparing Women and Men". Psychological Bulletin 129 (4): 569. 
Escobar-Lemmon, María y Kendall Funk. 2018. “Women's Representation in Subnational Governments: Replicating National Institutions or Forging a Unique Path?". En Gender and Representation in Latin America, editado por Leslie Schwindt-Bayer. New York: Oxford University Press, 99-120.

Escobar-Lemmon, María, y Michelle Taylor-Robinson. 2005. "Women Ministers in Latin American Government: When, Where and Why?". American Journal of Political Science 49 (4): 829-844.

Escobar-Lemmon, María, y Michelle Taylor-Robinson. 2009. “Getting to the Top: Career Paths of Women in Latin American Cabinets". Political Research Quarterly 62 (4): 685-699.

Escobar-Lemmon, María, y Michelle Taylor-Robinson. 2016. Women in Presidential Cabinets: Power Players or Abundant Tokens? New York: Oxford University Press.

Franceschet, Susan, Claire Annesley y Karen Beckwith. 2017. "What do Women Symbolize? Symbolic Representation and Cabinet Appointments". Politics, Groups, and Identities 5 (3): 488-493.

Franceschet, Susan, Mona Lenna Krook y Jennifer Piscopo. 2012. "Themes and Implications for Future Research on Gender Quotas". En The Impact of Gender Quotas, editado por Susan Franseschet, Mona Lenna Krook y Jennifer Piscopo.. Oxford: Oxford University Press, 229-242.

Franceschet, Susan, y Jennifer Piscopo, 2014. "Sustaining Gendered Practices? Power, parties, and Elite Political Networks in Argentina". Comparative Political Studies 47 (1): 85-110.

Franceschet, Susan, y Jennifer Piscopo. 2008. "Gender Quotas and Women's Substantive Representation. Lessons from Argentina". Politics \& Gender 4 (3): 393-425.

Franceschet, Susan. 2011. "Gender Policy and State Architecture in Latin America". Politics \& Gender 7(2): 273-279.

Granara, Aixa. 2014. "Representación legislativa de las mujeres en las provincias argentinas, 1989-2011". América Latina Hoy 66: 115-143.

Hinojosa, Magda. 2012. Selecting Women, Electing Women: Political Representation and Candidate Selection in Latin America. Philadelphia: Temple University Press.

Holman, Mirya R. 2014. Women in Politics in the American City. Philadelphia: Temple University Press.

Holman, Mirya R. 2018. "Women in Local Government: What We Know and Where We Go from Here". State and Local Government Review 49 (4): 285-296.

Htun, Mala y Mark Jones. 2001. “Engendering the Right to Participate in Decision-Making: Electoral Quotas and Women's Leadership in Latin America". En Gender and the Politics of Rights and Democracy in Latin America, editado por Nikki Craske y Maxine Molyneux. London: Palgrave, 32-56.

Htun, Mala. 2003. "Women and Democracy". En Constructing Democratic Governance in Latin America, $2^{\text {nd }}$.Edition. editado por Jorge Domínguez y Michael Shifter. Baltimore: Johns Hopkins University Press, 118-136.

Inglehart, Ronald, y Pipa Norris. 2003. Rising Tide: Gender Equality and Cultural Change around the World. Cambridge: Cambridge University Press.

Jacob, Suraj, John A. Scherpereel y Melinda Adams. 2014. “Gender Norms and Women's Political Representation: A Global Analysis of Cabinets, 1979-2009". Governance 27 (2): 321-345.

Jalalzai, Farida. 2016. Women Presidents of Latin America: Beyond Family Ties?. New York: Routledge Press.

Katz, Jonathan, y Gary King. 1999. "A Statistical Model for Multiparty Electoral Data". American Political Science Review 93 (1): 15-32.

Krook, Mona Lenna, y Diana O'Brien. 2012. “All the President's Men? The Numbers and Portfolio Allocations of Female Cabinet Ministers". Journal of Politics 74 (3): 840-855.

Liu, Shan-Jan Sarah y Lee Ann Banaszak. 2017. "Do Government Positions Held by Women Matter? A Cross-National Examination of Female Ministers' Impacts on Women's Political Participation". Politics \& Gender 13 (1): 132-162. 
Lopreite, Debora. 2014. "Explaining Policy Outcomes in Federal Contexts: The Politics of Reproductive Rights in Argentina and Mexico". Bulletin of Latin American Research 33 (4): 389-404.

Matland, Richard. 1998. "Women's Representation in National Legislatures: Developed and Developing Countries". Legislative Studies Quarterly 23 (1): 109-125.

Micozzi, Juan Pablo. 2013. “Ellos los representantes, nosotros los representados. Reflexiones sobre la representación y la conexión electoral en la Argentina contemporánea". Revista SAAP (7) 2: 401-411.

Morgan, Jana, y Melissa Buice. 2013. "Latin American Attitudes toward Women in Politics: The Influence of Elite Cues, Female Advancement, and Individual Characteristics". American Political Science Review 107 (4): 644-662.

Norris, Jeanne M. y Anne M. Wylie. 1995. "Gender Stereotyping of the Managerial Role Among Students in Canada and the United States". Group \& Organization Management 20 (2): 167-182.

Norris, Pipa. 1997. Passages to Power: Legislative Recruitment in Advanced Democracies. Cambridge: Cambridge University Press.

O'Brien, Diana Z., Matthew Mendez, Jordan Carr Peterson y Jihyun Shin. 2015. "Letting Down the Ladder or Shutting the Door: Female Prime Ministers, Party Leaders, and Cabinet Members". Politics \& Gender 11 (4): 689-717.

O'Brien, Diana. 2015. "Rising to the Top: Gender, Political Performance, and Party Leadership in Advanced Articles Industrial Democracies". American Journal of Political Science 59 (4): 1022-1039.

Papke, Leslie E. y Jeffrey M. Wooldridge. 1996. "Econometric Methods for Fractional Response Variables with an Application to 401 (k) Plan Participation Rates". Journal of Applied Econometrics 11 (6): 619-632.

Reyes-Housholder, Catherine. 2016. "Presidentas Rise: Consequences for Women in Cabinets?". Latin American Politics \& Society 58 (3): 3-25.

Reynolds, Andrew. 1999. "Women in the Legislatures and Executives of the World: Knocking at the Highest Glass Ceiling". World Politics (51) 4: 547-572.

Schwindt-Bayer, Leslie. 2010. Political Power and Women's Representation in Latin America. Oxford: Oxford University Press.

Siaroff, Alan. 2000. "Women's Representation in Legislatures and Cabinets in Industrial Democracies". International Political Science Review 21 (2): 197-215.

Smith, Heidi Jane M. y Keith D. Revell. 2016. “Micro-Incentives and Municipal Behaviour: Political Decentralization and Fiscal Federalism in Argentina and Mexico". World Development 77: 231-248.

Smulovitz, Catalina. 2015. "Legal Inequality and Federalism: Domestic Violence Laws in the Argentine Provinces". Latin American Politics and Society 57 (3): 1-26.

Thiébault, Jean Louis. 1991. "The Social Background of Western European Cabinet Ministers". En The Profession of Government Minister in Western Europe, editado por Jean Blondel y Jean-Louis Thiébault. New York: Palgrave Macmillan, 19-30.

Tomz, Michael, Joshua A. Tucker y Jason Wittenberg. 2002. “An Easy and Accurate Regression Model for Multiparty Electoral Data". Political Analysis 10 (1): 66-83.

Wylie, Kristin y Pedro Dos Santos. 2016. "A Law on Paper Only: Electoral Rules, Parties, and the Persistent Underrepresentation of Women in Brazilian Legislatures". Politics $\mathcal{E}$ Gender 12 (3): 415-442. 
Tiffany D. Barnes es doctora (PhD) en Ciencia Política por la Rice University (EE. UU.) y realizó un posdoctorado en el Kellog Institute Kellogg Institute for International Studies en la University of Notre Dame (EE. UU.). Actualmente es profesora asociada en la University of Kentucky (EE. UU.). Es autora del libro Gendering Legislative Behavior: Institutional Constraints and Collaboration, editado por Cambridge University Press, el cual obtuvo en 2017 el Premio Alan Rosenthal de la American Political Science Association (APSA). Ha publicado también en American Journal of Political Science; Journal of Politics; Comparative Political Studies; Political Research Quarterly; Politics \& Gender; Governance; Election Law Journal; Journal of Women, Politics \& Policy; PS: Political Science \& Politics; Conflict Management and Peace Science; y en The Journal of Political Science. Sus investigaciones obtuvieron financiamiento de la fundación National Science Foundation, la beca Ora N. Arnold Fellowship y de la University of Kentucky College of Arts and Sciences, obteniendo varios reconocimientos por sus trabajos académicos, entre los que destacan: el premio Sophonisba Breckinridge Award de la Midwest Political Science Association, el premio Marian Irish Award en 2017 y el premio Southern Political Science Association, Early Career Award de la Midwest Women's Caucus for Political Science. Correo electrónico: tiffanydbarnes@uky.edu

Tomás Ciocci es candidato a doctor en Ciencia Política y magister en la misma disciplina por la Universidad Torcuato Di Tella (Argentina), además de licenciado en Ciencia Política por la Universidad Nacional de La Matanza (Argentina). Actualmente es becario del Consejo Nacional de Investigaciones Científicas y Técnicas (Conicet) y dicta clases de Introducción a la Estadística e Instituciones Políticas Comparadas en la Universidad Nacional de la Matanza. Ha presentado trabajos de investigación en diversos congresos latinoamericanos de Ciencia Política y desarrollado cursos de postgrado sobre Metodología en Brasil y Uruguay. Su agenda de investigación se concentra en la política subnacional en Argentina, analizando intención de voto, participación de mujeres y fortaleza de los gobernadores. Correo electrónico: tomas.cioccipardo@mail.utdt.edu

Débora Lopreite es doctora (PhD) en Políticas Públicas por la Carleton University (Canadá), magister scientiarium en Administración Pública y licenciada en Ciencia Política por la Universidad de Buenos Aires (Argentina). Ha sido investigadora postdoctoral en el Departamento de Ciencia Política de la The Université du Québec à Montréal (Canadá). Actualmente es profesora en la Universidad de Buenos Aires y se desempeña como investigadora y asesora en el Instituto Nacional de las Mujeres (INAM). Ha enseñado cursos de grado y postgrado sobre Políticas Públicas, Género y Desarrollo Internacional en la Carleton University, la Université d'Ottawa (Canadá), la Universidad de Buenos Aires, la Facultad Latinoamericana de Ciencias Sociales-Sede Académica Argentina (FLACSO-Argentina, la Universidad Nacional de Tres de Febrero (Argentina) y la Universidad Nacional de Río Negro (Argentina). Sus investigaciones han sido financiadas por el Consejo Canadiense de Ciencias Sociales y Humanidades (Sshrc) y el Centro para la Investigación sobre Desarrollo Internacional (IDRC). Ha publicado artículos en revistas internacionales como Nueva Sociedad, Global Social Policy, Latin American Perspectives, Social Politics y Bulletin of Latin American Research, además de capítulos en volúmenes editados por la University of British Columbia-UBC Press (Canadá), McGill-Queen's University Press (Canadá) y University of Toronto Press. Correo electrónico: dlopreite@sociales.uba.ar 